\title{
Effective stresses and estimations of the apparent Biot coefficient in stacked clay nanolayers
}

\author{
T. HONORIO* $\dagger$, L. BROCHARD*and M. VANDAMME*
}

\begin{abstract}
WORD COUNT: 1996
In materials with nanometric pores, such as clays, confinement effects alter the way the pressure of fluids is transferred to the solid. Even for liquid-saturated porous materials with poorly compressible solid skeleton, this can translate into apparent Biot coefficients (defined as the partial derivative of the stress orthogonal to the clay layer with respect to the bulk pressure of water at fixed volume) of adsorbing materials being higher than 10 or even negative. In this article, molecular simulations of saturated clays layers are presented. From these simulation, the apparent Biot coefficient is derived as a function of the basal spacing. It is observed that the strains of an infinite stack of clay layers are generically not governed by the Terzaghi effective stress; but apparent Biot coefficients remain in the range 0 to 1 for stable basal spacings larger than $1.5 \mathrm{~nm}$. For the sake of comparison, results of molecular simulations of a confined Lennard-Jones fluid are also provided. In this case, Biot coefficients vary from a negative value up to more than 15 according to the pore size.
\end{abstract}

KEYWORDS: saturated clays; molecular scale; effective stress; confined fluids

ICE Publishing: all rights reserved

\section{NOTATION}

- $b$ apparent Biot coefficient

- $d$ basal spacing

- $d^{*}=d / \sigma_{L J}$ reduced basal spacing

- $N$ number of values of confining pressure computed at each basal spacing and temperature

- $N_{w}$ number of fluid molecules

- $P$ confining pressure

- $P_{0}$ is the least square estimate of Y-intercept of $P\left(P_{w}\right)$

- $P_{w}$ pressure of water

- $P^{*}=P \sigma^{2} / \varepsilon_{L J}$ reduced confining pressure

- $P_{w}^{*}=P_{w} \sigma^{2} / \varepsilon_{L J}$ reduced bulk pressure of the fluid

- $r_{f f}$ distance between fluid molecule

- $r_{f s}$ shortest distance between the fluid molecule and the solid wall

- $S$ entropy

- $T$ temperature

- $U_{f f}$ and $U_{f s}$ energy of interaction in 12-6 and 9-3 Lennard-Jones potential, respectively.

- $V$ volume

\footnotetext{
Manuscript received...

Published online at www.geotechniqueletters.com

i*Université Paris-Est, Laboratoire Navier (UMR 8205), CNRS, ENPC, IFSTTAR, 6 \& 8 Avenue Blaise Pascal, 77455 Marne-laVallée, France

†Current address: Université Paris-Est Créteil, Laboratoire Modélisation et Simulation Multi-Echelle, MSME UMR 8208 CNRS, 94010, Créteil cedex, France; tulio.honorio-de-faria@upec.fr
}

- $\Delta_{b}$ standard deviation of $b$

- $\varepsilon_{L J}$ Lennard-Jones parameter with dimensions of energy

- $\mu_{w}$ chemical potential of water

- $\Pi$ disjoining pressure

- $\sigma$ total external stress

- $\sigma_{t}$ Terzaghi effective stress

- $\sigma_{L J}$ Lennard-Jones parameter with dimensions of length

- $\langle$.$\rangle average operator$

\section{INTRODUCTION}

The concept of effective stress introduced by Terzaghi (1936) is widely employed in geomechanics. The Terzaghi effective stress $\sigma_{t}$ is the difference between the total external stress $\sigma$ and the pressure of water $P_{w}$ :

$$
\sigma_{t}=\sigma-P_{w}
$$

This concept is generally assumed valid despite the hierarchical character of pore sizes in clay-rich soils and rocks. As sketched in Figure 1, clays have a multiscale microstructure with hierarchical pore size distribution. The basic structural units of clays are thin $(\sim \mathrm{nm})$ mineral layers. The ordering of those sheets, isomorphic substitutions as well as the different types of ions in pore solution lead to the variety of clays found in nature. The mineral layers are locally organized in stacks. The space in-between mineral layers can be filled by water in smectites due to their moderate structural charge (Meunier 2005). The 


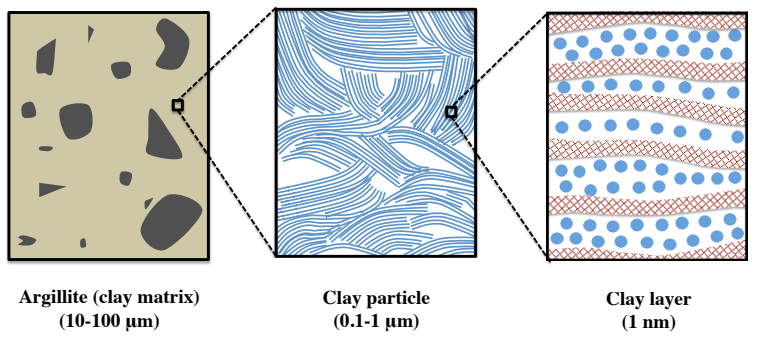

Fig. 1. Multiscale microstructure of clays. Micropores are identified at the layer scale (right), mesopores at the clay particle scale (center), and macropores at the clay matrix scale (left).

characteristic size of the pores at that scale is on the order of the size of water molecules.

About $90 \%$ of the porosity in clays refers to pores smaller than $50 \mathrm{~nm}$ (e.g. Cariou (2010)). The assumptions and state equations of poromechanics are generally based on bulk thermodynamics. However, at the nanoscale fluid-solid molecular interactions become significant formulations not accounting for interface effects may not hold. Fluid molecules in micropores (pores with a diameter $<2 \mathrm{~nm}$ ) which interact with the atoms of the solid are said confined or adsorbed and exhibit physical behaviors that differ from the bulk. Additionally, since the size of atoms and molecules is not negligible with respect to the associated length scale of the pore, even basic concepts such as pore size become ambiguous at the nanoscale.

The apparent Biot coefficient $b$ is defined as follows:

$$
b=\left.\frac{\partial \sigma}{\partial P_{w}}\right|_{V}
$$

Whenever the concept of Terzaghi effective stress holds, the apparent Biot coefficient is strictly 1. Research on simple confined fluids (Brochard et al. (2012)) shows that due to interfacial effects $b$ can be larger than 1 or even negative. One can reasonably wonder whether strains at the scale of clay layers are still governed by the Terzaghi effective stress.

In the absence of interfacial effects, Coussy (2010) shows that Terzaghi effective stress principle holds whenever the solid skeleton is poorly compressible and the pores are saturated. At the nanoscale however, due to interfacial effects, even for rigid solid layers and saturated pores, apparent Biot coefficients different from 1 can appear as discussed in the following.

Here, it is reported molecular simulations results of saturated clays layers from which the apparent Biot coefficient is derived as a function of the basal spacing. The clay studied is a montmorillonite with sodium interlayer ions (noted $\mathrm{Na}-\mathrm{Mmt}$ ). The present study is limited to the case of saturated clays, i.e. the water pressure is greater than the saturation vapor pressure of water at a given temperature. For the sake of comparison, results for a Lennard-Jones fluid confined in a slit pore are also provided.

\section{MATERIALS AND METHODS}

Molecular simulations of realistic clay systems and of a Lennard-Jones (LJ) fluid confined in-between $\mathrm{LJ}$ walls are performed. In both cases, the simulations are performed in grand canonical ensemble for the fluid (i.e. the volume $V$ and the temperature $T$ of the entire system and the chemical potential of the fluid $\mu_{w}$ are fixed; whereas the pressure $P$ and entropy $S$ of the entire system as well as the number of fluid molecules $N_{w}$ fluctuate). At a given temperature, note that an imposed $\mu_{w}$ corresponds to an imposed fluid pressure $P_{w}$ since at constant temperature the chemical potential of water $\mu_{w}=f\left(P_{w}, T\right)$ is a function of the fluid pressure. This thermodynamic ensemble mimics the drained condition encountered in many geotechnical applications. For both systems (Na-Mmt and $\mathrm{LJ}$ ), the ranges of temperatures and pressures of fluid were chosen so that the bulk fluid is in its liquid state (saturated conditions). Moreover, in all simulations, the solid layers are kept rigid (incompressible). The total pressure $P$ is computed from virial expression as in Allen and Tildesley (1989). Note that the space and time averages of the virial stresses converges to Cauchy stress tensor (Zhou 2003). The solid layers are interacting via long-range electrostatic forces that are disturbed by electrostatics and depletion effects due electrolyte confinement, these interactions lead to the difference between $P$ and $P_{w}$.

Molecular simulations of Na-Mmt are detailed in Honorio et al. (2017). Here, the results at $300 \mathrm{~K}$ and $500 \mathrm{~K}$ considered in Honorio et al. (2017) are recalled and new results at $400 \mathrm{~K}$ and other imposed water pressures $P_{w}$ at $300 \mathrm{~K}$ and $500 \mathrm{~K}$ are also presented. The elemental composition of the montmorillonite studied is: $\mathrm{Na}_{6}^{+} \cdot\left[\mathrm{Si}_{62} \mathrm{Al}_{2}\right]\left[\mathrm{Mg}_{4} \mathrm{Al}_{28}\right] \mathrm{O}_{160}(\mathrm{OH})_{32} \cdot \mathrm{nH}_{2} \mathrm{O}$. The isomorphic substitutions are randomly located in the octahedral and tetrahedral sheets. The location of these substitutions is reported to induce only minor effects on thermodynamic results Ngouna et al. (2014). ClayFF force field (Cygan et al. 2004) and SPC/E water model (Berendsen et al. 1987) are employed. Simulations are performed with Towhee (Martin 2013). A slit pore is considered and series of simulations are run for different basal spacings $d$ (i.e., center-to-center distance of clay layers). Figure 2 displays snapshots of equilibrium configurations of Na-Mmt in $0 \mathrm{~W}, 1 \mathrm{~W}$ and $2 \mathrm{~W}$ states.

Additionally, a 2D LJ system is studied, in which the interactions are described by a 12-6 and 9-3 Lennard-Jones potentials, respectively:

$$
\begin{aligned}
& U_{f f}(r)=4 \varepsilon_{L J}\left[\left(\frac{\sigma_{L J}}{r_{f f}}\right)^{12}-\left(\frac{\sigma_{L J}}{r_{f f}}\right)^{6}\right] \\
& U_{f s}(r)=\varepsilon_{L J}\left[\frac{2}{15}\left(\frac{\sigma_{L J}}{r_{f s}}\right)^{9}-\left(\frac{\sigma_{L J}}{r_{f s}}\right)^{3}\right]
\end{aligned}
$$

where $r_{f f}$ is the distance between fluid molecule, $r_{f s}$ is the shortest distance between the fluid molecule and the solid wall, $\varepsilon_{L J}$ and $\sigma_{L J}$ are the LJ parameters with dimensions of energy 
OW

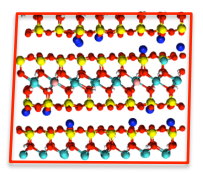

1W

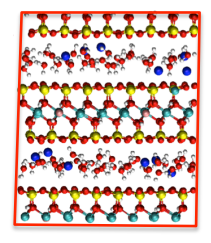

$2 \mathrm{~W}$

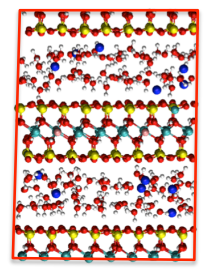

Fig. 2. Snapshot of the configurations of $\mathrm{Na}-\mathrm{Mmt}$ at $300 \mathrm{~K}$ under a water pressure $P_{w}$ of $49 \mathrm{MPa}$ in $0 \mathrm{~W}, 1 \mathrm{~W}$ and $2 \mathrm{~W}$ states, i.e. with 0,1 and 2 layers of interlayer water, respectively (the corresponding basal spacings are $d=9.55,12.75$ and $15.15 \AA$, respectively). A projection in the ac plane of the triclinic periodic super-cell is shown. Atoms are colored as follows: $\mathrm{Mg}$, pink; $\mathrm{Na}$, blue; $\mathrm{Si}$, yellow; $\mathrm{O}$, red; $\mathrm{H}$, white; $\mathrm{Al}$, green.

and length, respectively. Simulations are run with LAMMPS (Plimpton et al. 1995) and further detailes are provided in Brochard et al. (2017).

\section{RESULTS}

Figure 3 shows the confining pressure isotherms of $\mathrm{Na}-\mathrm{Mmt}$ at 300,400 and $500 \mathrm{~K}$ for five different water pressures $P_{w}$. The confining pressure $P$ is the normal pressure acting on solid clay layers ( $z$-direction here) $P(d)=-\sigma_{z z}$ and oscillates as function of the basal spacing $d$. For each curve, $P_{w}$ is estimated from the average of the computed $P$ corresponding to $d>20$ $\AA$. This definition of $P_{w}$ is necessary due to the difficulty in identifying the precise $\mu_{w}$ corresponding to a target pressure $P_{w}$. Note that for $d>20 \AA, P$ only fluctuates around an average value.

Confining pressure isotherms have an oscillating aspect due to the structuration of fluids in small pores. Each oscillation $n$ corresponds to a hydration state $n W$, i.e., the fluid is structured in $n$ layers within the pore. Clays generally exhibit oscillations corresponding to one $(1 W)$, two $(2 W)$ and three $(3 W)$ water layers. $0 \mathrm{~W}$ refers to the dehydrated state and the "pore water" state is called $\infty \mathrm{W}$. In Figure 3, $0 \mathrm{~W}$ state corresponds to the first branch decreasing with $d$, the first oscillation corresponds to $1 W$ and the second oscillation to $2 W$. Oscillations related to subsequent hydration states could not be directly identified with the accuracy of the molecular simulations. However, since the hydration states are separated by the average diameter of a water molecule, the basal spacings corresponding to $3 W$ and subsequent hydration states can be approximately determined.

As detailed in Brochard et al. (2017), the basal spacings between the defined hydration states are unstable, i.e. they are not observed at the layer scale when the confining pressure is controlled. Only the portions of confining pressure isotherms that decrease with the basal spacing are stable (recall that, in a thermodynamic equilibrium, a state is stable if the pressure does not increases with volume). The system at the scale of a clay particle (or stack of layers) may however assume a deformation corresponding to an average basal spacing $\langle d\rangle=$ $d_{u}$, where $d_{u}$ is a basal spacing in the unstable domain. This deformation is reached by mixing of stable states within the particle, as detailed in Honorio et al. (2017).

Following Honorio et al. (2017), confining pressure isotherms can be also studied in terms of disjoining pressure $\Pi$ defined as the difference between the confining pressure $P(d)$ and the water pressure $P_{w}$ :

$$
\Pi=P(d)-P_{w}
$$

Here, the disjoining pressure isotherms obtained at any given $P_{w}$, at a given temperature, almost overlap with each other when plotted versus basal spacing. The differences between the $\Pi$ isotherms are not significant with respect to the amplitude of the oscillations associated to $1 \mathrm{~W}$ and $2 \mathrm{~W}$ states. Also, these differences are on the order of the fluctuations associated with the simulations. Therefore, $\Pi$ seems to depend only on the basal spacing $d$ and on the temperature $T$, but not on the water pressure $P_{w}$.

The results in Figure 3 are used to estimate the apparent Biot coefficient of clay layers at a given temperature as a function of the basal spacing $d$ as shown in Figure 4. Large fluctuations of $b$ are observed, which is expected provided the fluctuations in the confining pressure isotherms. Fluctuations of the apparent Biot coefficient are smaller at high temperatures. The red rectangles depict the stable domains corresponding to a confining pressure in the range $P_{w} \pm 50 \mathrm{MPa}$. Considering these domains only, apparent Biot coefficients approximately remain within the range between 0 and 1 .

For basal spacings larger than $15 \AA(3 W)$ at $500 \mathrm{~K}$, the apparent Biot coefficient can be fairly approximated by 1 . For $0 W, 1 W$ and portions of $2 W$ states, $b$ fluctuates and is on average less than 1 . Therefore, deformations of clay layers, due to interfacial effects, are not necessarily governed by the Terzaghi effective stress when the interlayer contains 2 layers of water molecules or less and at lower temperatures.

Confining pressure isotherms and apparent Biot coefficients are also obtained for the confined LJ fluid. In Figure 5 (a), it can be observed that the confining pressure isotherms of confined LJ fluid also exhibit oscillations, but with more clearly defined hydration states thanks to more accurate results. Again, the portions for which the pressure increases with the basal spacing are unstable. For a given reduced basal spacing $d^{*}=d / \sigma_{L J}$, the reduced confining pressure $P^{*}=$ $P \sigma_{L J}^{2} / \varepsilon_{L J}$ evolves linearly with the reduced bulk pressure of the fluid $P_{w}^{*}=P_{w} \sigma_{L J}^{2} / \varepsilon_{L J}$ (Figure 5 (b)). The apparent Biot coefficient (i.e. the slope of these lines) is therefore constant with $P_{w}$. In Figure 5 (c) it can be observed that the apparent Biot coefficient is strongly dependent on the basal spacing $d$ and can reach both very high values ( $b=15.4$ for $d^{*}=1.575 \AA$ ) and negative values $\left(b=-0.61\right.$ for $d^{*}=2.025 \AA$ ). These large variations can be found in both stable and unstable domains. This simple example proves that, due to interfacial effects, the pressure in the fluid is not directly tranferred to the solid, 

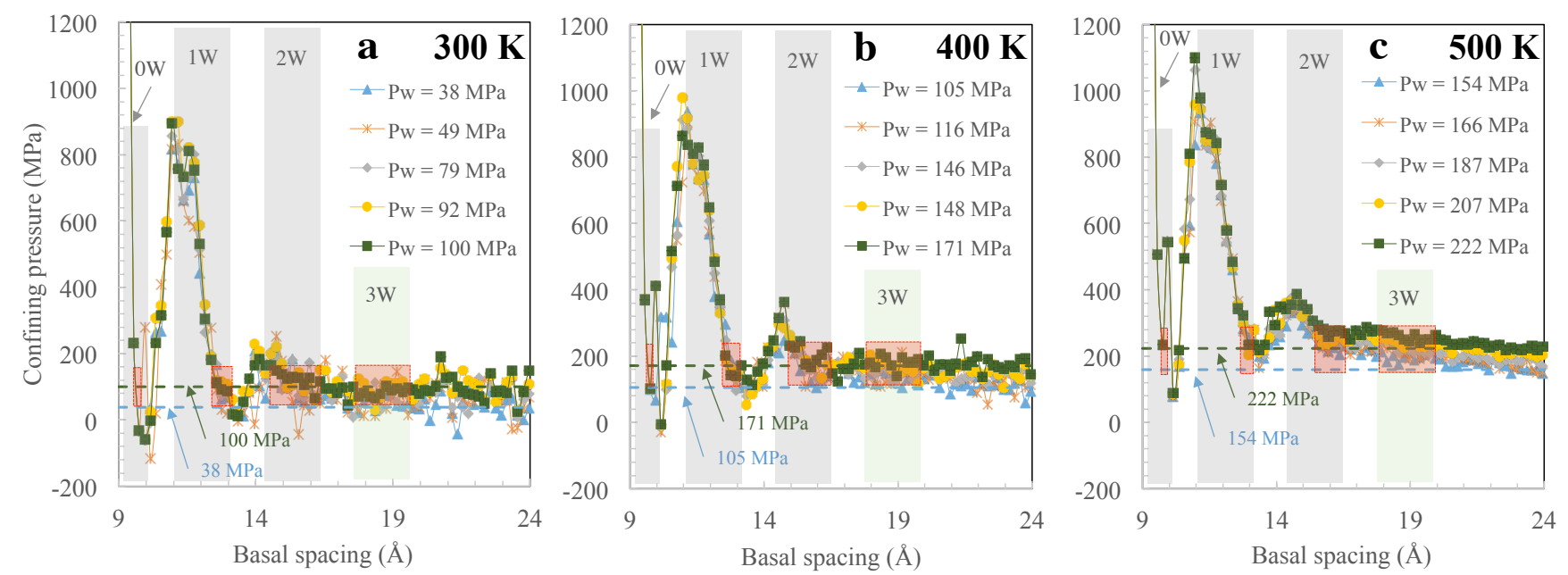

Fig. 3. Confining pressure isotherms of Na-Mmt at a 300, b 400 and c $500 \mathrm{~K}$ as a function of the basal spacing and water pressures $P_{w}$. The dashed lines depict the minimum and maximum values of the imposed water pressures $P_{w}$. The stable portions corresponding to $0 W, 1 W$ and $2 W$ states are represented by the gray rectangles; the range associated with $3 W$ (light yellow rectangle) is estimated accounting for the average diameter of a water molecule of circa $3.55 \AA$ (i.e. the LJ radius of oxygens in SPC/E model).
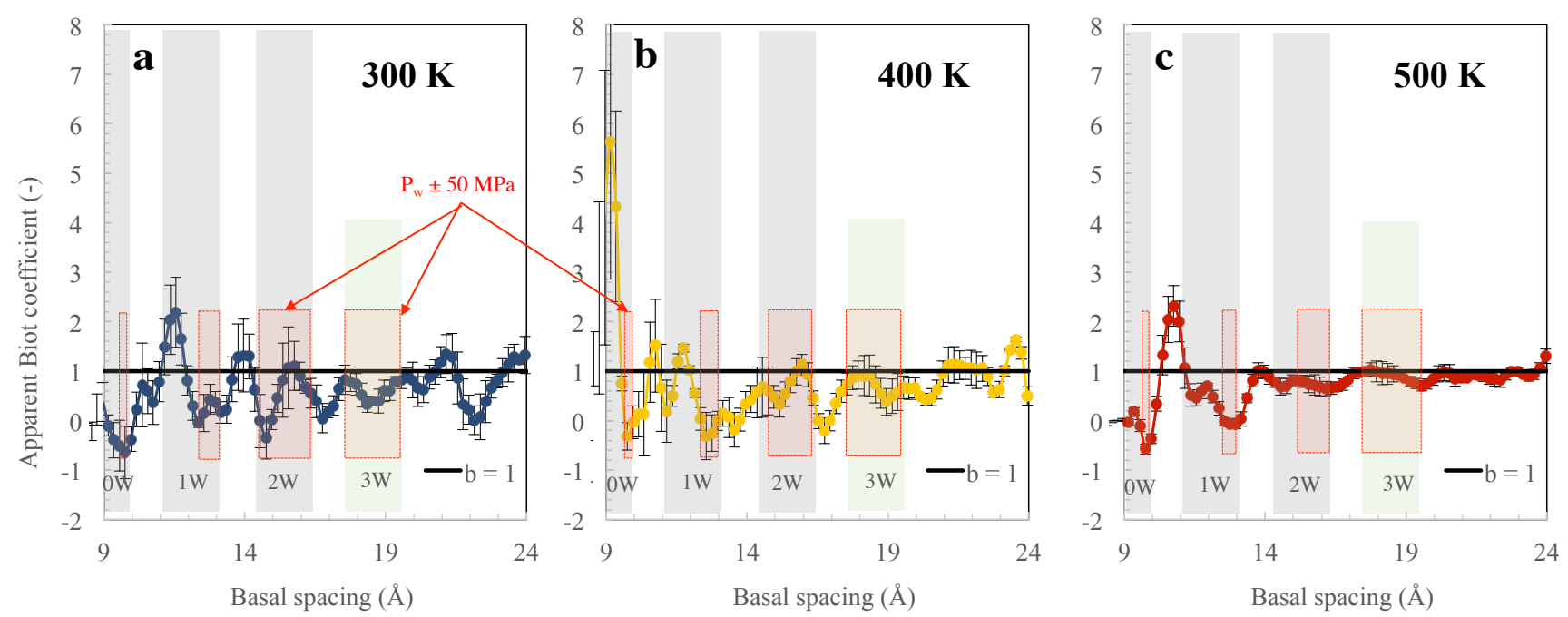

Fig. 4. Apparent Biot coefficients of Na-Mmt at a 300, b 400 and c $500 \mathrm{~K}$ as a function of the basal spacing $d$. The apparent Biot coefficient $b$ is obtained as the least square estimate of the linear regression of $P$ with respect to $P_{w}$. Savitzky-Golay (Roth 2000) filter is applied to the confining pressure as a function of $d$. The standard deviation $\Delta b$ of $b$ is estimated from the propagation of errors by the formula (Taylor 1997): $\Delta b=\Delta P \sqrt{\frac{N}{N \sum P_{w}^{2}-\left(\sum P_{w}\right)^{2}}}$, where $N$ is the number of values of confining pressure computed at each basal spacing and temperature; and $\Delta P=\sqrt{\frac{1}{N-2} \sum\left(P-P_{0}-b P_{w}\right)^{2}}$ is the uncertainty of the confining pressure $\left(P_{0}\right.$ is the least square estimate of the $\mathrm{Y}$-intercept of $\left.P\left(P_{w}\right)\right)$. The red rectangles are portions of the stable domains corresponding to a confining pressure in the range $P_{w} \pm 50 \mathrm{MPa}$. Simulations at lower temperature are expected to produce results with more variability since water molecules might not have enough kinetic energy (directly related to the temperature) to escape from some configurations associated with local energy minimum. Since a GCMC simulation is basically insertion/deletion of water molecules, in hydration states with less water, the difference of one single molecule impacts non-negligibly the results which can also translate in more variability.

following the concept of effective stresses. This is true even for an incompressible solid saturated by a liquid.

Compared to the LJ system, clay layers are subjected to the particularities of water behavior (Brochard and Honorio 2017), ion correlations and heterogeneity of clay layers (e.g. granularity and isomorphic substitutions). One may attribute to these aspects the reasons why less strong variations in apparent Biot coefficient are observed in clay layers than in the LJ system. This hypothesis would need further investigations.
Other molecular-scale informed studies (Le et al. 2013) (Mainka et al. 2016) resort to Density Functional Theory (DFT) and Mean Spherical Approximations (MSA) to compute the disjoining pressure in clays. Contrary to molecular simulations, analyses based on DFT/MSA and PoissonBoltzmann distribution cannot capture the non-monotonous character of counterions distribution within clay micropores (Tinnacher et al. 2016). Here, an explicit electrolyte accounting directly for dipole, ion and clay surface (cross-)interactions 

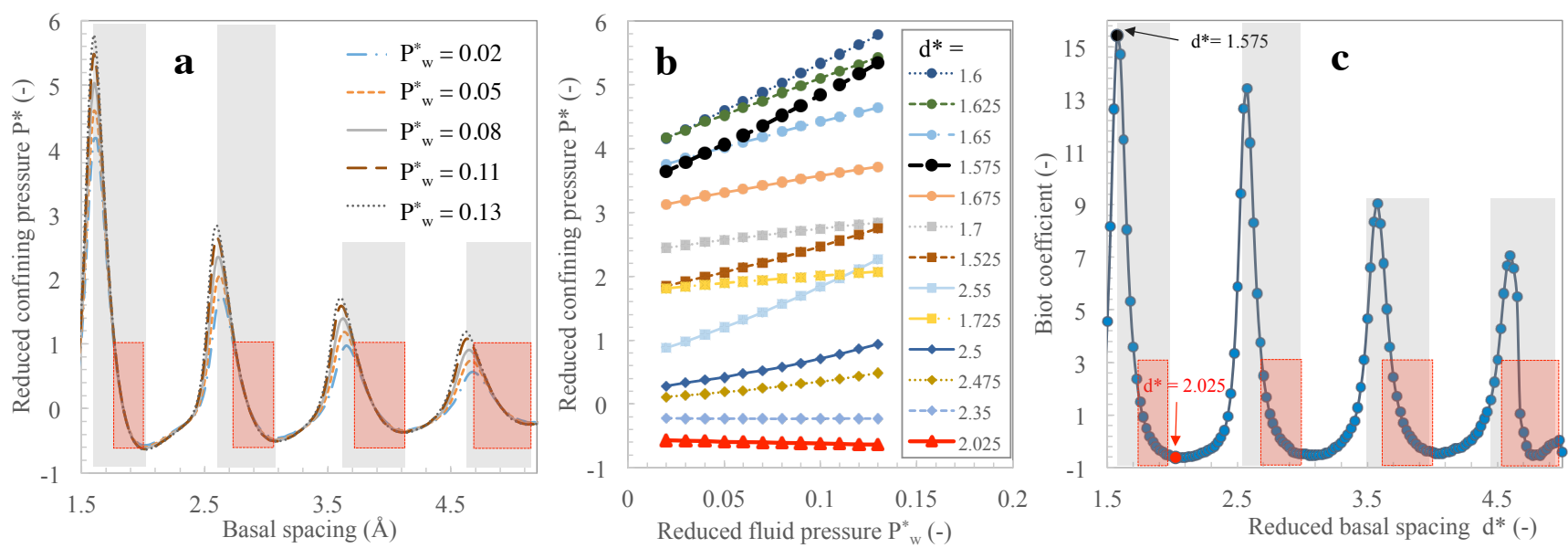

Fig. 5. a Confining pressure isotherms of LJ fluid between 9-3 LF walls in $2 \mathrm{D}$ as a functions of the basal spacing for different pressures of fluid reservoir $P^{*}$. The stable portions are identified by the gray rectangles (only for the curve with $P^{*}=0.13$, for the sake of clarity). The red rectangles are portions of the stable domains corresponding to a confining pressure in the range $P^{*}<1$. $\mathbf{b}$ Confining pressure of $\mathrm{LJ}$ fluid as a function of $P^{*}$ for some fixed basal spacings $d$. c Apparent Biot coefficient as a function of the basal spacing $d$. The corresponding stable portions are also shown.

(including aspects related to the preferential adsorption sites on layers rough surface (Underwood et al. 2016)) was considered.

\section{CONCLUSION}

In this article, it was showed that interfacial effects alter the way the pressure of fluids is transferred to the solid phases in materials with nanometric pores, even if the solid remains poorly compressible. This is clear in simulations of confined simple fluids. In realistic molecular simulation of clays, interfacial effects are also manifest, but apparent Biot coefficients remain in the range 0 to 1 for stable basal spacings larger than $1.5 \mathrm{~nm}$. These results limit the validity of analysis not coping with interfacial effects (such as the original Terzaghi effective stress principle) at the clay layer nanoscale to only some specific conditions. This is not inconsistent with experimental observations at the macroscale, since micropores is just one class of pores in clay-rich materials (Cariou (2010)). Considering that larger pores present apparent Biot coefficients equal to 1, after upscaling, the effective (macroscopic) Biot coefficient must be close to 1 (provided that, as observed here, the apparent Biot coefficients of micropores remains close to $1)$.

\section{ACKNOWLEDGEMENTS}

Funding through the project TEAM2ClayDesicc from the French national Research Agency (Agence Nationale de la Recherche contract ANR-14-CE-05-0023-01) is gratefully acknowledged.

\section{REFERENCES}

Terzaghi, K. (2003). The shearing resistance of saturated soils and the angle between the planes of shear, International Conference on Soil Mechanics and Foundation Engineering, 4th edn. Addison-Wesley.
Meunier, A. (2005). Clays, Springer Science \& Business Media.

Cariou, S. (2010). Couplage hydro-mcanique et transfert dans largilite de Meuse/Haute-Marne: approches exprimentale et multi-chelle, $\mathrm{PhD}$ Thesis. Ecole des Ponts ParisTech.

Brochard, L., Vandamme, M., Pellenq, R.J.-M. (2012). Poromechanics of microporous media, Journal of the Mechanics and Physics of Solids 60, 606622. doi:10.1016/j.jmps.2012.01.001

Coussy, O. (2012). Mechanics and Physics of Porous Solids, Wiley.

Allen, M.P., Tildesley, D.J. (1989). Computer Simulation of Liquids, Wiley.

Zhou, M. (2003). A new look at the atomic level virial stress: on continuum-molecular system equivalence, Proceedings of the Royal Society of London A: Mathematical, Physical and Engineering Sciences 459, 23472392. doi:10.1098/rspa.2003.1127

Honorio, T., Brochard, L., Vandamme, M. (2017). Hydration phase diagram of clay layers and particles from molecular simulations, Langmuir 33, 1276612776. doi:10.1021/acs.langmuir.7b03198

Ngouana W., B.F., Kalinichev, A.G., (2014). Structural Arrangements of Isomorphic Substitutions in Smectites: Molecular Simulation of the Swelling Properties, Interlayer Structure, and Dynamics of Hydrated CsMontmorillonite Revisited with New Clay Models, J. Phys. Chem. C 118, 1275812773. doi:10.1021/jp500538z

Cygan, R.T., Liang, J.-J., Kalinichev, A.G. (2004). Molecular Models of Hydroxide, Oxyhydroxide, and Clay Phases and the Development of a General Force Field, J. Phys. Chem. B 108, 12551266. doi:10.1021/jp0363287

Berendsen, H.J.C., Grigera, J.R., Straatsma, T.P., (1987). The missing term in effective pair potentials, J. Phys. Chem. 91, 62696271. doi:10.1021/j100308a038

Martin, M.G. (2013). MCCCS Towhee: a tool for Monte Carlo molecular simulation, Molecular Simulation 39, 12121222. doi:10.1080/08927022.2013.828208

Plimpton, S. (1995). Fast Parallel Algorithms for Short-Range Molecular Dynamics, Journal of Computational Physics 117, 119. doi:10.1006/jcph.1995.1039

Brochard, L., Honorio, T., Vandamme, M., Bornert, M., Peigney, M. (2017). Nanoscale origin of the thermo-mechanical behavior of 
clays, Acta Geotech. 119. doi:10.1007/s11440-017-0596-3

NIST (2014). SAT-TMMC: Liquid-Vapor coexistence properties SPC/E Water, [WWW Document]. http://www.nist.gov/

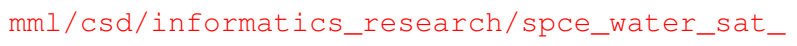
Irc.cfm.

Bird, P. (1984). Hydration-phase diagrams and friction of montmorillonite under laboratory and geologic conditions, with implications for shale compaction, slope stability, and strength of fault gouge, Tectonophysics 107, 235260. doi:10.1016/0040-1951(84)90253-1

Roth, A. (2000). Savitzky-Golay Smoothing Filters, Mathematica program. Max-Planck-Institut fr Medizinische Forschung.

Taylor, J. (1997). Introduction to Error Analysis, the Study of Uncertainties in Physical Measurements, 2nd Edition. Published by University Science Books, 648 Broadway, Suite 902, New York, NY 10012, 1997.

Brochard, L., Honorio, T. (2017). Anomalous thermal expansion of confined water in clay, (submitted paper).

Le, T.D., Moyne, C., Murad, M.A., Lima, S.A. (2013). A two-scale non-local model of swelling porous media incorporating ion size correlation effects, Journal of the Mechanics and Physics of Solids 61, 24932521. doi:10.1016/j.jmps.2013.07.012

Mainka, J., Murad, M.A., Tran, V.D., Moyne, C. (2016). Application of a Multi-Scale form of Terzaghis Effective Stress Principle for Unsaturated Expansive Clays to Simulate HydroMechanical Behavior During Hydration, in: E-UNSAT 2016. doi:10.1051/e3sconf/20160914024

Underwood, T., Erastova, V., Greenwell, H.C. (2016). Ion Adsorption at Clay-Mineral Surfaces: The Hofmeister Series for Hydrated Smectite Minerals, Clays and Clay Minerals 64, 472487. doi: 10.1346/CCMN.2016.0640310

Tinnacher, R.M., Holmboe, M., Tournassat, C., Bourg, I.C., Davis, J.A. (2016). Ion adsorption and diffusion in smectite: Molecular, pore, and continuum scale views, Geochimica et Cosmochimica Acta 177, 130149. doi: 10.1016/j.gca.2015.12.010 